\title{
Matrix of Constraints for the Motion of the Planar Kinematic Chains with Rotational Links with Clearances
}

\author{
Jan-Cristian Grigore ${ }^{1 *}$, Alexandru Jderu ${ }^{2}$, Marius Enachescu ${ }^{2}$ \\ ${ }^{1}$ University of Piteşti, Piteşti, 110040, Romania \\ ${ }^{2}$ University POLITEHNICA of Bucharest, Bucharest, 060042, Romania \\ *Corresponding author: jan_grigore@yahoo.com
}

\begin{abstract}
Our goal is to present a general approach for the planar chains with rotational links with clearances. This approach is realized in a multibody style, the main problem being the determination of the matrix of constraints. Introduction

The application of the multibody type methods [1-13], made possible the elaboration of certain general algorithms for the numerical, dynamical and elasto-dynamical calculation of the kinematical and dynamical parameters for the mechanical systems' motions. These algorithms can be applied both to the determined kinematical mechanical systems with and without friction $[4,9,14]$, respectively with one degree of mobility, and to the systems with one degree of mobility and a single motor element as, for instance, $[2,7,12]$, the mechanical convertor of torque created by G. Constantinescu. The applications of the multibody type methods for the studying of the planar mechanical systems having articulations with clearances (rotational kinematical joints) [3,4,6,10,14], assumes the inserting of certain virtual without mass elements, which leads to singular matrix of inertia and, consequently, it does not permit the separation of the general system of equations in two systems from which result, in order, the time history of the reactions and then the time history of the kinematical parameters. In this paper we elaborate a multibody type method based on a new form of the matrix of constraints, method that permits the numerical dynamical study of the planar systems with rotational joints with or without clearance, with one or several degrees of freedom.
\end{abstract}

\section{General Aspects}

We consider the planar kinematical chain from the Figure 1 at which the elements denoted by $1,2, \ldots$ are linked one to another by rotational kinematical links with or without clearance $\mathrm{O}_{1}, \mathrm{O}_{2}$ Denoting by $C_{i}$ the centre of weight of an element $i$, which is either a bar, or a shell, and denoting by $C_{i} x_{i} y_{i}$ the proper reference system, Figure 2, then the position of this elements, relative to the general fixed reference system $O X Y$, is defined by the coordinates $X_{i}, Y_{i}$ of the centre of weight, and by the angle $\theta_{i}$ between the axes $C_{i} x_{i}$ and $O X$.
This element, linked to the next element $j$ by the rotational joint $O_{k}$, can have a point $\widetilde{O}_{l}\left(\widetilde{X}_{l}, \widetilde{Y}_{l}\right)$ with known motion,

$$
\widetilde{X}_{l}=\widetilde{X}_{l}(t), \widetilde{Y}_{l}=\widetilde{Y}_{l}(t) .
$$

If the rotational kinematical joint $O_{k}$ is with clearance, Figure 3, with permanent contact between the elements $i$ and $j$, then the clearance can be defined by the difference $\quad r_{k}=O_{k}^{(i)} O_{k}^{(j)}, r_{k}=r_{k}^{(i)}-r_{k}^{(j)}$, between the radius $r_{k}^{(i)}$ of the rim and the radius $r_{k}^{(j)}$ of the shaft, and by the angle $\alpha_{k}$ between $O_{k}^{(i)} O_{k}^{(j)}$ and $O X$. 


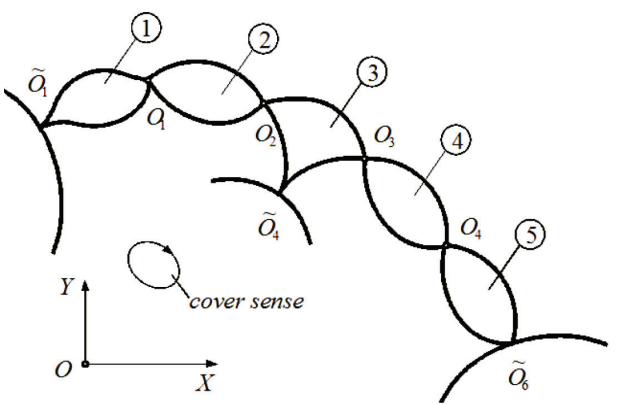

Figure 1. Planar kinematical chain.

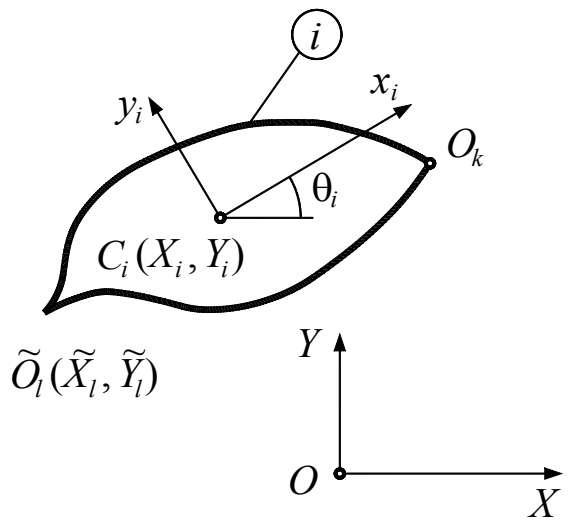

Figure 2. Determination of position for the element $i$.

Functions of constraint. Conditions of constraint. Matrix of constraints

Conditions given by the existence of the rotational kinematical joint

We consider the elements $i, j$, Figure 4 linked one to another by the rotational kinematical joint with clearance $O_{k}$ and let $x_{k}^{(i)}, y_{k}^{(j)}$ be the coordinates of the point $O_{k}^{(i)}$ in the local reference system $C_{i} x_{i} y_{i}$. If we use the notations

$U_{k X}^{(i)}=x_{k}^{(i)} \cos \theta_{i}-y_{k}^{(i)} \sin \theta_{i}$

$U_{k Y}^{(i)}=x_{k}^{(i)} \sin \theta_{i}+y_{k}^{(i)} \cos \theta_{i}$,

then the coordinates $X_{k}^{(i)}, Y_{k}^{(i)}$ of the point $O_{k}^{(i)}$ in the reference system $O X Y$ are expressed by the relations

$X_{k}^{(i)}=X_{i}+U_{k X}^{(i)}, Y_{k}^{(i)}=Y_{i}+U_{k Y}^{(i)}$, and the coordinates $X_{k}^{(j)}, Y_{k}^{(j)}$ of the point $O_{k}^{(j)}$ in the system $O X Y$ are obtained with the same relations (2) in which we replace the index $i$ by the index $j$.

Keeping into account the sense cover sense of a contour, Figure 4, and using the notation

$\left\{\mathbf{D}_{k}\right\}=\frac{1}{r_{k}}\left[\begin{array}{l}X_{k}^{(j)}-X_{k}^{(i)} \\ Y_{k}^{(j)}-Y_{k}^{(i)}\end{array}\right]$

one can write the constraining functions in the form, equations number (4)

$\left\{\left\{\mathbf{D}_{k}\right\}=\{\mathbf{0}\}\right.$, if $O_{k}$ is a kinematical joint without clearance $\left\{\left\{\mathbf{D}_{k}\right\}^{T}\left\{\mathbf{D}_{k}\right\}-1=0\right.$, if $O_{k}$ is a kinematical joint with clearance

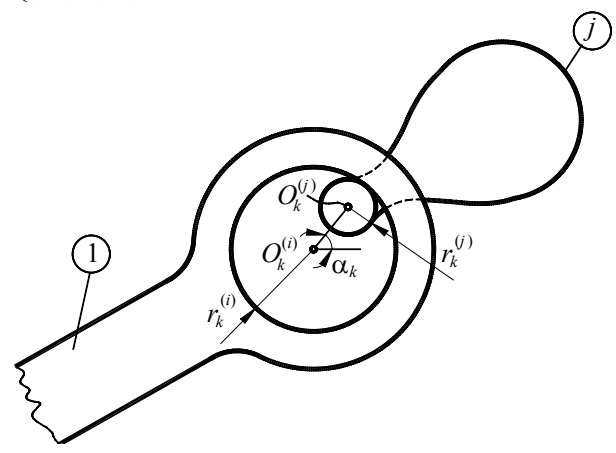

a) constructive schema

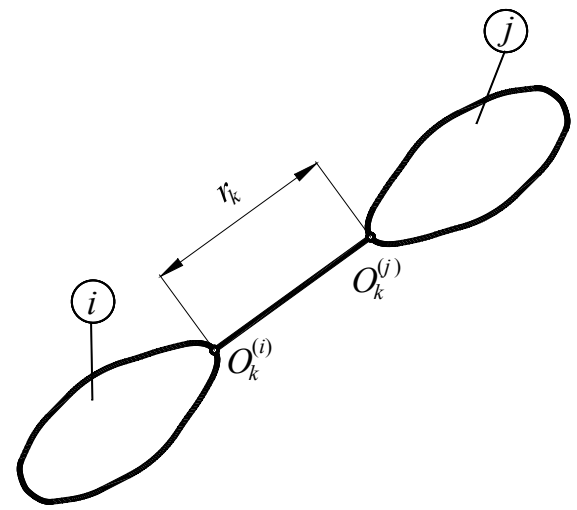

b) kinematical schema.

Figure 3. Rotational kinematical joint with clearance 


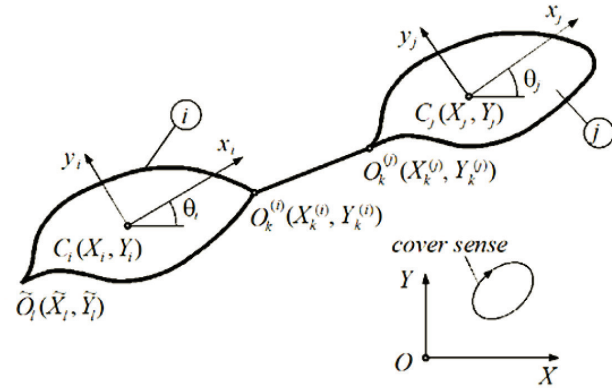

Figure 4. The elements $i, j$ and the rotational kinematical joint with clearance $O_{k}$.

By derivation of the relations (1), (3) in respect to time and keeping into account the notations

$\left[\mathbf{B}_{k}^{(i)}\right]=\left[\begin{array}{ccc}1 & 0 & -U_{k y}^{(i)} \\ 0 & 1 & U_{k x}^{(i)}\end{array}\right]$

$\left[\mathbf{E}_{k}^{(i)}\right]=\left\{\mathbf{D}_{k}\right\}^{T}\left[\mathbf{B}_{k}^{(i)}\right]$

$\left\{\mathbf{q}_{i}\right\}=\left[\begin{array}{lll}X_{i} & Y_{i} & \theta_{i}\end{array}\right]^{T}$

one deduces the equalities

$\dot{U}_{k X}^{(i)}=-\dot{\theta}_{i} \cdot U_{k Y}^{(i)}, U_{k Y}^{(i)}=\dot{\theta}_{i} \cdot U_{k X}^{(i)}$

$\left\{\dot{\mathbf{D}}_{k}\right\}=\frac{1}{r_{k}}\left[\mathbf{B}_{k}^{(j)}\right]\left\{\dot{\mathbf{q}}_{j}\right\}-\frac{1}{r_{k}}\left[\mathbf{B}_{k}^{(i)}\right]\left\{\dot{\mathbf{q}}_{i}\right\}$

In these conditions, by the derivation of the relations (4) in respect to time, one obtains the conditions of constraint, we can write the relation (7).

$$
\left\{\begin{array}{c}
{\left[\mathbf{B}_{k}^{(j)}\right]\left\{\dot{\mathbf{q}}_{j}\right\}-\left[\mathbf{B}_{k}^{(i)}\right]\left\{\dot{\mathbf{q}}_{i}\right\}=\{\mathbf{0}\},} \\
\text { if } O_{k} \text { is a kinematical joint without clearance } \\
{\left[\mathbf{E}_{k}^{(j)}\right]\left\{\dot{\mathbf{q}}_{j}\right\}-\left[\mathbf{E}_{k}^{(i)}\right]\left\{\dot{\mathbf{q}}_{i}\right\}=0,} \\
\text { if } O_{k} \text { is a kinematical joint with clearance }
\end{array}\right.
$$

In the case of the rotational kinematical joint with clearance, Figure 4, using the angle $\alpha_{k}$, we can write the relation

$\left\{\mathbf{D}_{k}\right\}=\left[\begin{array}{ll}\cos \alpha_{k} & \sin \alpha_{k}\end{array}\right]^{T}$

and from here results for the matrix $\left[\mathbf{E}_{k}^{(i)}\right\rfloor$ the expression (9);

$\left[E_{k}^{(1)}\right]=$

$=\left[\begin{array}{lll}\cos \alpha_{k} & \sin \alpha_{k} & -x_{k}^{(i)} \sin \left(\theta_{i}-\alpha_{k}\right)-y_{k}^{(i)} \cos \left(\theta_{i}-\alpha_{k}\right)\end{array}\right]$
3.2. Conditions given by the existence of a point $\widetilde{O}_{l}$ with known motion

In this case, denoting by $\tilde{x}_{l}, \tilde{y}_{l}$ the coordinates of the point $\widetilde{O}_{l}$ in the reference frame $C_{i} x_{i} y_{i}$ and using the notations $\widetilde{U}_{l X}^{(i)}=\widetilde{x}_{l}^{(i)} \cos \theta_{i}-\widetilde{y}_{l}^{(i)} \sin \theta_{i}$, $\widetilde{U}_{l Y}^{(i)}=x_{l}^{(i)} \sin \theta_{i}+y_{l}^{(i)} \cos \theta_{i}$, $\left[\widetilde{\mathbf{B}}_{l}^{(i)}\right]=\left[\begin{array}{ccc}1 & 0 & -\widetilde{U}_{l Y}^{(i)} \\ 0 & 1 & \widetilde{U}_{l X}^{(i)}\end{array}\right],\left\{\widetilde{\mathbf{F}}_{l}\right\}=\left[\begin{array}{cc}\dot{\widetilde{X}}_{l} & \dot{\tilde{Y}}_{l}\end{array}\right]^{T}$, one obtains the equalities $X_{i}+\widetilde{U}_{l X}^{(i)}=\widetilde{X}_{i}$, $Y_{i}+\widetilde{U}_{l Y}^{(i)}=\widetilde{Y}_{i}$,

which, by derivation in respect to time and keeping into account the relations $\dot{\widetilde{U}}_{l x}^{(i)}=-\dot{\theta}_{i} \cdot \widetilde{U}_{l Y}^{(i)}, \dot{\widetilde{U}}_{l Y}^{(i)}=\dot{\theta}_{i} \cdot \widetilde{U}_{l X}^{(i)}$, lead us to the constraining condition

$\left[\widetilde{\mathbf{B}}_{l}^{(i)}\right]\left\{\dot{\mathbf{q}}_{i}\right\}=\left\{\widetilde{\mathbf{F}}_{l}\right\}$

\subsection{Matrix of constraints. Matrix equation of constraint}

Matrix of constraints is obtained by assembling matrices of $\left.\left\lfloor\mathbf{B}_{k}^{(i)}\right\rfloor, \mid \mathbf{E}_{k}^{(i)}\right\rfloor$, and $\left[\widetilde{\mathbf{B}}_{l}\right]$ type met in the relations (9), (10), relations which are written for each rotational kinematical joint, respectively for each point with known motion. Thus, for the kinematical chain drawn in the Figure 5 , at which the element 1 has uniform rotational motion with the known angular

(7) speed $\omega$, and the rotational kinematical joint $\mathrm{O}_{3}$ is one with clearance, one obtains the matrix of constraints,

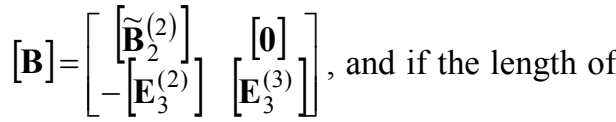
the element 1 is equal to $l_{1}$, then the coordinates of the point $\mathrm{O}_{2}$ in the general reference system $O_{1} X Y$ are $\widetilde{X}_{2}=l_{1} \cos \omega t$ , $\widetilde{Y}_{2}=l_{1} \sin \omega t$ and we get the matrix equations of constraining $[\mathbf{B}]\{\dot{\mathbf{q}}\}=\{\mathbf{C}\}$, 
where $\{\mathbf{q}\}=\left[\begin{array}{llllll}X_{2} & Y_{2} & \theta_{2} & X_{3} & Y_{3} & \theta_{3}\end{array}\right]^{T}$ ,$\{\mathbf{C}\}=\left[\begin{array}{lll}-l \omega \sin \omega t & -l \omega \cos \omega t \quad 0\end{array}\right]^{T}$.

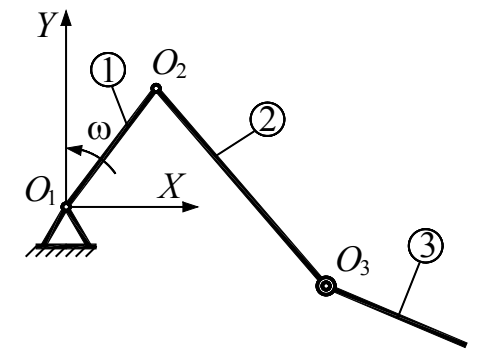

Figure 5. Kinematical chain with the element $\mathrm{O}_{1} \mathrm{O}_{2}$ in rotational motion and the rotational

kinematical joint $\mathrm{O}_{3}$ with clearance

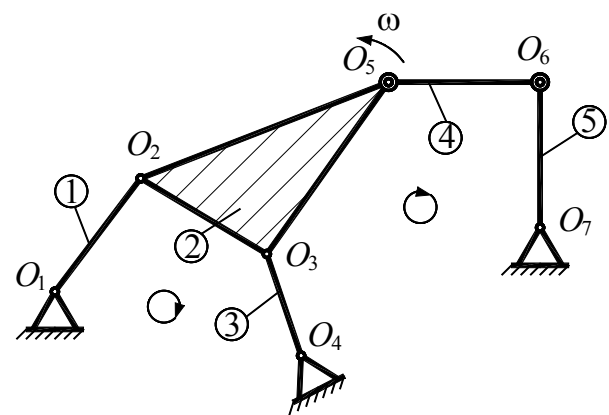

Figure 6. System with five elements and the rotational kinematical joints $\mathrm{O}_{5}, \mathrm{O}_{6}$, with clearance.

In the case when the element 1 has not a determined motion, one obtains the matrix of constraints

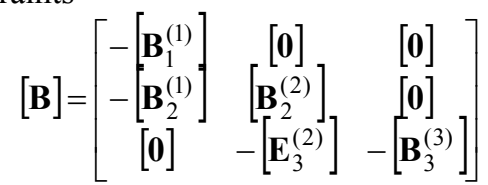

and the matrix equation of constraints is $[\mathbf{B}]\{\dot{\mathbf{q}}\}=\{\mathbf{0}\}$, where $\{\mathbf{q}\}=\left[\begin{array}{lllllllll}X_{1} & Y_{1} & \theta_{1} & X_{2} & Y_{2} & \theta_{2} & X_{3} & Y_{3} & \theta_{3}\end{array}\right]^{T}$.

Analogously, for the system with five elements drawn in the Figure 6, with the rotational kinematical joint $\mathrm{O}_{5}, \mathrm{O}_{6}$ with clearance, we obtain the matrix of constraints

$$
[\mathbf{B}]=\left[\begin{array}{ccccc}
{\left[\mathbf{B}_{1}^{(1)}\right]} & {[\mathbf{0}]} & {[\mathbf{0}]} & {[\mathbf{0}]} & {[\mathbf{0}]} \\
-\left[\mathbf{B}_{2}^{(1)}\right] & {\left[\mathbf{B}_{2}^{(2)}\right]} & {[\mathbf{0}]} & {[\mathbf{0}]} & {[\mathbf{0}]} \\
{[\mathbf{0}]} & -\left[\mathbf{B}_{3}^{(2)}\right] & {\left[\begin{array}{l}
\left.\mathbf{B}_{3}^{(3)}\right] \\
{[\mathbf{0}]}
\end{array}\right][\mathbf{0}]} & {[\mathbf{0}]} & {[\mathbf{0}]} \\
\left.\mathbf{B}_{4}^{(3)}\right] & {[\mathbf{0}]} & {[\mathbf{0}]} \\
{[\mathbf{0}]} & -\left[\mathbf{E}_{5}^{(2)}\right] & {[0]} & -\left[\mathbf{E}_{5}^{(4)}\right. \\
{[\mathbf{0}]} & {[\mathbf{0}]} & {[\mathbf{0}]} & -\left[\mathbf{E}_{6}^{(4)}\right. \\
{[\mathbf{0}]} & {[\mathbf{0}]} & {[\mathbf{0}]} & {[\mathbf{0}]} & {\left[\begin{array}{l}
{[\mathbf{0}]} \\
\mathbf{E}_{6}^{(5)} \\
\mathbf{B}_{7}^{(5)}
\end{array}\right]}
\end{array}\right.
$$

and the matrix equation of the constraints $[\mathbf{B}]\{\dot{\mathbf{q}}\}=\{\mathbf{0}\}, \quad$ where $\{q\}=\left[\begin{array}{lllllll}X_{1} & Y_{1} & \theta_{1} & \ldots & X_{5} & Y_{5} & \theta_{5}\end{array}\right]^{T}$.

3.4. Derivative of the matrix of constraints with respect to time

From the relations (5), (6), in the conditions (7), (8), result;

$$
\begin{aligned}
& {\left[\dot{\mathbf{B}}_{k}^{(i)}\right]=-\dot{\theta}_{i} \cdot\left[\begin{array}{ccc}
0 & 0 & U_{k X}^{(i)} \\
0 & 0 & U_{k Y}^{(i)}
\end{array}\right]} \\
& {\left[\dot{\tilde{\mathbf{B}}}_{l}^{(i)}\right]=-\dot{\theta}_{i} \cdot\left[\begin{array}{ccc}
0 & 0 & \widetilde{U}_{l X}^{(i)} \\
0 & 0 & \widetilde{U}_{k Y}^{(i)}
\end{array}\right]} \\
& {\left[\dot{\mathbf{E}}_{k}\right]=\frac{1}{r_{k}}\left\{\dot{\mathbf{q}}_{j}\right\}^{T}\left[\mathbf{B}_{k}^{(j)}\right]^{T}\left[\mathbf{B}_{k}^{(i)}\right]-\frac{1}{r_{k}}\left[\mathbf{B}_{k}^{(j)}\right]^{T \cdot}\left[\mathbf{B}_{k}^{(i)}\right]+\left\{\mathbf{D}_{k}\right\}^{T}\left[\dot{\mathbf{B}}_{k}^{(i)}\right]}
\end{aligned}
$$

The derivative of the matrix $[\mathbf{B}]$ with respect to time is obtained using these expressions.

\section{Conclusions}

The establishing in the paper of a new form of the matrix of constraints made possible the elaboration of the multibody method that permits both the numerical study of the general motion of the planar jointed systems with clearances, and the numerical study of the equilibrium positions.

The numerical applications solved here confirm the statements mentioned above.

\section{Acknowledgement}

This work was supported by Romanian Ministry of Education and by Executive Agency for Higher Education, Research, 
Development and Innovation Funding, under project ENIAC 03/2013.

\section{References}

14) Amirouche, F., Fundamentals of multibody dynamics. , Birkhänser, Boston, Berlin, (2004).

15) Constantinescu, G., Teoria sonicității, Editura Academiei R.S.R, București, (1985).

16) Erkaya, S., Uzmay, I., Investigation on effect of joint clearance on dynamics of four-bar mechanism. Nonlinear Dyn., 58, 179-198, (2009).

17) Flores, P., Ambrósio, J., Revolute joints with clearance in multibody systems. Comput. Struct. 82, 13591369, (2004).

18) Flores, P., Modeling and simulation of wear in revolute clearance joints in multibody systems. Mechanism and Machine Theory, 44, 1211-1222, (2009).

19) Grigore, J.-C., Contribuţii la studiul dinamic al mecanismelor cu jocuri. Teză de doctorat, Universitatea din Piteşti,(2008).

20) Pandrea, N., Calculul dinamic al convertorului mecanic de cuplu "G. Constantinescu” IFToMM Int. Symp.
SYROM 89 pag. 673-679, Bucharest, Romania,(1989).

21) Pandrea, N., Popa, D., Mecanisme. Editura Tehnică, București, (2000).

22) Penestri, E., Valentini, P., P., Vito, L., Multibody dynamics simulation of planar linkages with Dahl friction, Multibody Syst. Dyn. 17, 321-347, (2007).

23) Pfeiffer, F., Glocker, C., Multibody dynamics with unilateral contacts. Wiley, New York (1996).

24) Ravn, P., A continuous analysis method for planar multibody systems with joint clearance. Multibody Syst. Dyn. 2, 1-24, (1998).

25) Samanta, B., Mukherjee, A., Deb, K., Bond graph adaptive modular approach to analysis of planar mechanisms, World Congress Mechanisms and Machine Theory, vol. III, pag. 439-443, Sevilla, Spain, (1987).

26) ShabanA A., A., Dynamics of multibody systems. Cambridge University Press, Cambridge, 2005

Stoenescu, E., D., Marghitu, D.B., Dynamic analysis of a planar rigid-link mechanism with rotating slider joint and clearance, J. Sound Vib. 266, 394404, (2003). 